\title{
Web Content Authoring for Ubiquitous and Context Aware Learning - A Tag based Approach
}

\author{
Lopa Mandal \\ Jadavpur University \\ Kolkata, India
}

\author{
Pramatha Nath Basu \\ Jadavpur University \\ Kolkata, India
}

\begin{abstract}
Web pages are mostly designed keeping the desktop PCs in mind. Displaying huge web content into the small screen mobile devices helps the user in no way. Mobile learners, who need to learn across context with mobile devices, need only the essentials and very less explanation on the subject matter. To achieve this, the present workers propose a Mobile Tag Library to be used by web authors with other HTML tags to develop dual purpose web pages and also a mobile browser capable of selective retrieval of the corresponding portion of web content and display that in a small-screen device.
\end{abstract}

\section{General Terms}

Web Content Authoring, Mobile Tag Library, Mobile Browser, Web Browser, Tag

\section{Keywords}

Mobile learning, computer based web learning, Ubiquitous and context aware Learning, Mobile Tag, Mobile Browser

\section{INTRODUCTION}

Web documents have become an integral part of peoples' daily lives. It has opened up a window of immeasurable opportunities for information exchange, in connecting, sharing, and interacting among diverse groups of people. Of late there is a growing demand for viewing web pages on small screens of hand held devices. A handheld device equipped with a browser and a wireless connection can increase the utility of mobile phones tremendously by providing access to numerous information services, like study materials, travel guides, entertainment advice, latest news, flight schedules etc.. In such a situation technology enabled learning has received much attention with the newly developed devices or concepts. In this revolution of education system, advancement of mobile and wireless technology and reduction of cost of handheld devices can play a vital role to promote ubiquitous (anytime anywhere) mobile learning [1]. It means learning contents are available to the learner irrespective of time and location, whenever they need it.

Generally people use their handheld devices for the purpose of learning when they are not settled down in their classroom, studies or in front of a computer; rather, when they move from one context to another, switch locations, social groups, etc and learning takes place in the short gaps of these transitions. But learning in a multitasking environment is not always as productive as in case of situated learning due to considerable level of distraction. A research was conducted by a team from the Virginia Tech [2] to prove the extent to which mobile learners could be distracted while trying to learn using their mobile devices while going about other tasks. It was observed that the group that walked with a mobile device scored significantly less on an assessment than the group that sat at a desk with a computer. Considering the fact, short learning objects are found to be more suitable for the purpose of mobile learning [3]. For different such constraints mobile learning is generally not considered as the primary mode of education $[4,5]$. It is mostly considered as a complement to the existing traditional education system and a mode of informal learning $[6,7]$ providing a new range of possibilities to the students of the digital generation $[8,9,10]$.

Keeping the above mentioned issues in mind, the present work focuses on viewing selected portion of web-content using mobile devices in an acceptable manner, as and when required, to promote mobile $\&$ ubiquitous learning which can be especially helpful to the requirements of teachers [11] and working adults.

\section{EXISTING APPROACHES}

Technically, it has been possible to access the Internet on a mobile phone for several years already. However, the mobile browsing experience has often been cumbersome for users. The conversion of standard web pages to be used with these devices is a challenge, as the amount of content within each of the web pages is too large to be adequately displayed in the smaller display areas of handheld devices.

The problems that occur while browsing web content developed for PCs via mobile devices are as follows:

- the performance of web browsers on mobile devices is lower than that of PC browsers,

- the screen size of the mobile devices is very small,

- the operation of small buttons is cumbersome,

- huge content, that is suitable for learning using a desktop, is not suitable for mobile learning.

In recent years, various studies have been conducted on issues of web page manipulation for small-screen devices. In general, to resolve these issues, the idea was that content will be maintained separately for access by different devices, such as full HTML for desktop and laptop devices, HTML 3 or under for PDA devices and WAP or other formats for cell phones. It was very quickly realized that the cost of maintaining separate content bases can be enormous and it may not be a very good solution. In fact, limited web content is available for mobile phones as compared to that available 
for PCs. For the success of the mobile learning, it is important to ensure that the vast majority of content that is easily accessible on the web through standard desktop browsers is

also made available on mobile devices [12].

A web page usually contains various types of materials that are not related to the topic of the web-page, such as intra and inter hyperlinks to take the user to different part of a web page, logos etc. It can also contain multimedia objects such as image, audio, video and animation; games, use of flash technology, java scripts, image maps, Cascading Style Sheets (CSS) etc. A web page consists of various interaction contents like forms to collect user information or provide searching services. It contains various special words or paragraphs such as copyrights information that is not useful for many users. All these things have made the web a kaleidoscope of very interesting but complex content and layout. So there is a need to develop an approach so that the goal of an easy mobile web browsing can be achieved.

One of the existing conventions is to create and maintain the content for web and mobile separately. But as mentioned earlier, this is quite a costly affair, so only a few websites have their mobile version. The other approaches are transcoding $[13,14]$, automated re-authoring of web pages by rendering the tags and generating a page for mobile browser as needed. There are three major approaches of automated reauthoring which are: Table of Content [15], Summarization [16] and Hybrid [17]. The last concept combines the other two. These approaches can be applied on existing web pages. But due to the complex nature of the modern web pages, rendering becomes very difficult and sometimes the pages generated by this method, are not useful.

Study of standard scripting language shows that HTML is a scripting language for web while WML and XHTML MP are scripting languages for mobiles. Another scripting language SMIL (Synchronized Multimedia Integration Language) is an XML-based language that allows writing interactive multimedia presentation on Internet. It can display multiple file types (image, video, audio, text etc.) and can define duration and sequences of element as well as visibility and position of elements in the Internet presentation. SMIL is often used in the latest cellular phones. But there is no such tag defined in SMIL to selectively parse out the web content for mobile devices. Neither is there any scripting language using which content for both web browser and mobile browser can be developed simultaneously. So a new tag based solution is suggested here for extracting essential contents from web content for small screen devices. The assumptions are that a web page to be displayed in a mobile is mostly a subset of that displayed on a PC screen and some of the tags, meant for only mobile browser, are ignored by the web browsers whereas some HTML tags are ignored by the mobile browser. .

\section{PROPOSED SOLUTION}

\subsection{Selective extraction of web content for mobile display with special consideration to multimedia data}

In this work a Mobile Tag Library has been developed to be used by the web authors with other HTML tags while developing the web pages, to retrieve selected part of a web page, suitable to be displayed on small-screen handheld devices. These new tags give the author power as well as responsibility to specify which portion of the text or which web image will go to the mobile browser. These tags make the rendering process easy and help generating the mobile pages more accurate and useful. Using some tags of this tag library, authors can also specify which portion is only meant for mobile and not for web. This technique is especially useful for multimedia data. For example, authors can incorporate separate set of large images suitable for web learning using desktop PCs and set of small images, suitable for mobile browsers, which is of great advantage over all earlier web content manipulation techniques for handheld devices, as it does not require any image compression technique to convert large web images suitable for small screen devices. This selective extraction of web content for mobile devices is extremely effective for mobile learning, especially for the teachers or working adults who in certain situation need only a limited explanation at the point of need, instead of elaborate description on the subject matter.

\subsection{Sub-title based hierarchical display of content for improved mobile browsing experience}

The selected part of the web content will be displayed on the mobile browser in a sub-title based hierarchical approach on small-screen devices to improve the browsing experience on small screen devices.

In order to provide a new convention of extracting selected portion of the web content and easy browsing of mobile pages, an overview of the original web page is created. The overview is a systematic review and a List of Content for the original web page. Overview page is made by extracting the subtitles from the web page. These subtitles work as options on the overview page. When users select an option, the block under that subtitle opens. This overview page fits well into small screen of mobile phone. These blocks are obtained using subtitle based searching algorithm. So most important thing is segmentation of blocks. But there are two most important technical issues to segment a web page into various blocks. First how to detect a structure of a Web page and second is how to segment a web page into smaller blocks.

Block diagram of the mobile browser using this technique is described in Figure 1.

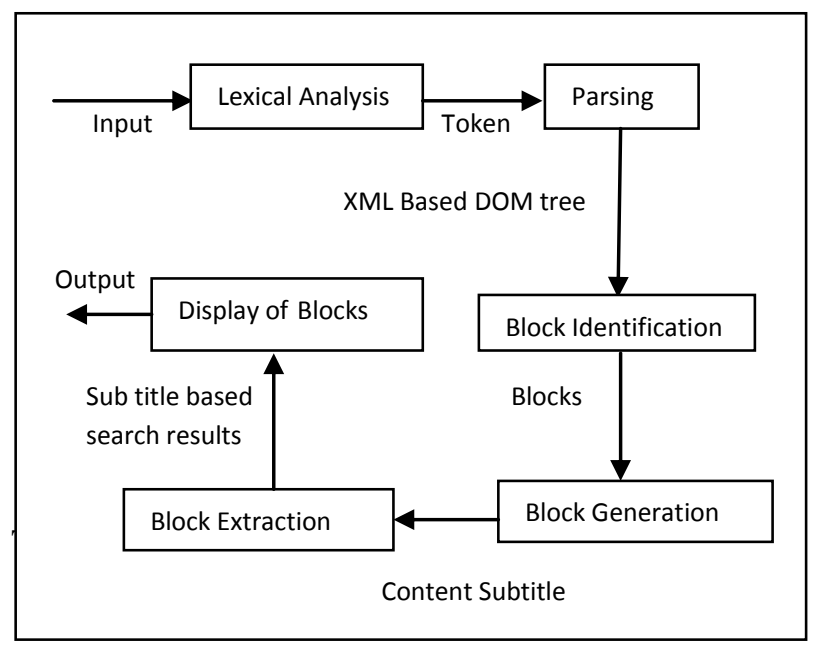

Fig 1: Block diagram of the proposed mobile page generation algorithm 
1. Lexical Analysis

2. Parsing

3 .Block identification

4. Block Generation

\section{Block Extraction}

\section{Display of Block}

Input of the lexical analysis is the original Web page, converted into XML document. At the time of analysis it includes start-tags, end-tags, and data. Output of the Lexical analysis is sequence of tokens. These tokens work as an input for the parser. Parsing transforms input text into an XML Based DOM tree by analyzing a sequence of tokens. Identification of block depends on the properties of DOM node itself and the children of the DOM node. It takes DOM tree as an input. For each and every node same block identification algorithm is applied until we get all selected blocks of web page for displaying on the mobile phone. The blocks are identified by a set of tags used by the author while developing the content for dual display. After identification of the block, a tag based selection is done to get the subtitle of that block. Block generation basically links subtitles to the block under that subtitle. The links are made up of the subtitles of each block added as list items in the list of contents on the overview page. These list items work as options to get the contents under that subtitle. Block extraction means after displaying the subtitle based overview page on the mobile phone, user will click on a subtitle option for getting the appropriate block and then only the requested block under that subtitle is extracted. This is the last step of the algorithm. After using top down approach of this algorithm if all the steps succeed, requested block will be displayed on the mobile phone. In this model, there can be any number of abstractions, but practical considerations dictate that anything more than two levels is confusing for most users [18]. So in this work, it has been restricted only up to two levels. So while developing the web page using this approach, authors have to design the content keeping this consideration in mind.

Eventually, according to the above algorithm, to define a portion of the web content as a block and to select some portion of that block, some tags have been designed which will work as tokens for the XML parser to generate the expected output. Figure 2 shows some of the tags of the proposed tag library and their usage along with other existing HTML tags. An example web page created by using those tags along with the HTML tags and the mobile display generated from that web page according to the above algorithm are shown in Figure 3,4,5,6 and 7 respectively.

\section{CONCLUSION}

In the present work a mobile tag library has been designed which is to be used along with the HTML tags by the web authors while developing the web pages. This would help to retrieve selected part of a web page, suitable to be displayed on small-screen handheld devices. The tag library also provides tags to send text and images which are only for the mobile and not for the web, giving the web author enough scope for simultaneous development of site material suitable for web browsers and as well as mobile browser to facilitate situated web learning (using normal-size computer screen) and mobile learning in respective context. The ability to incorporate multimedia data e.g. images, exclusively for

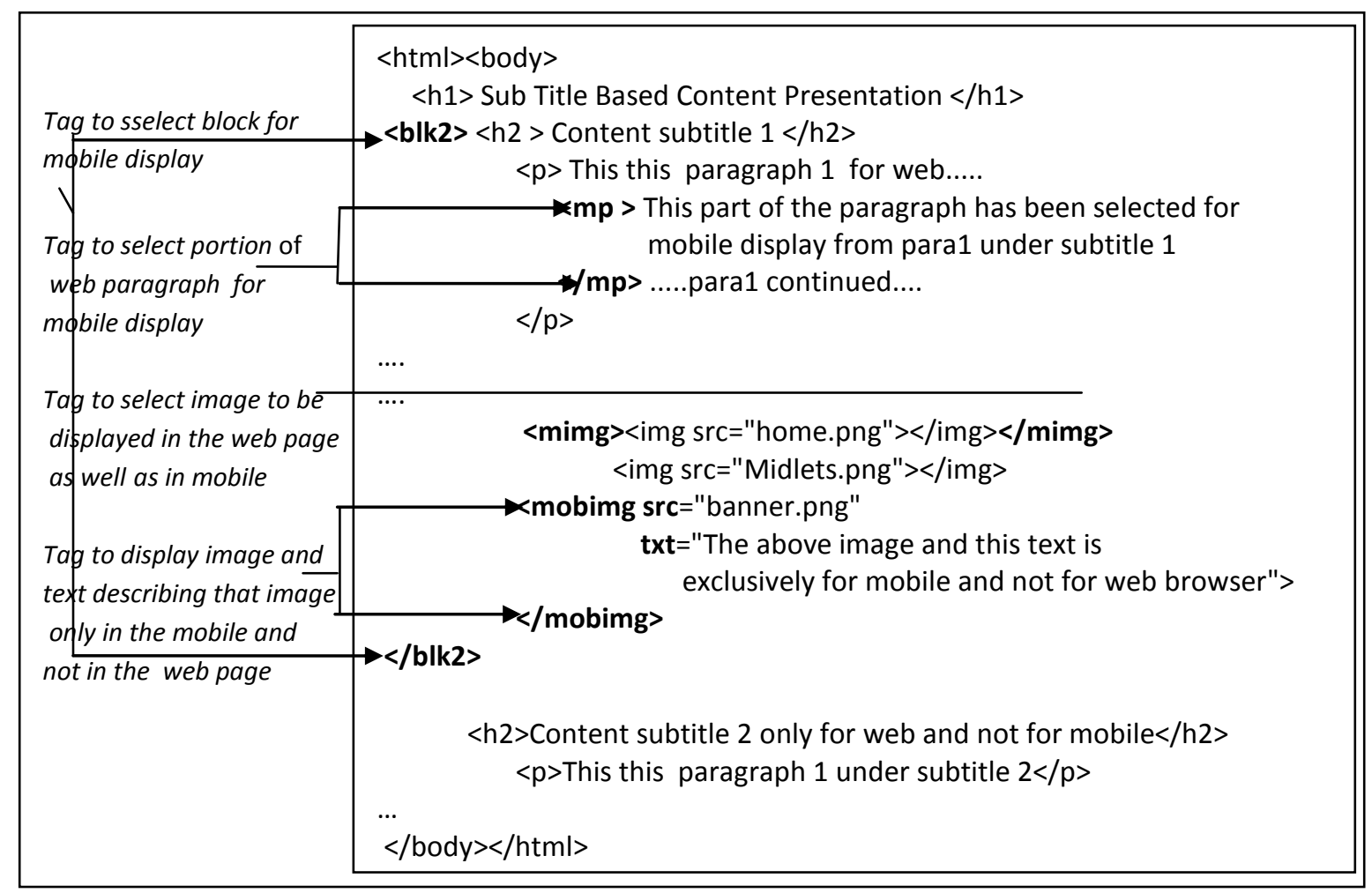

Fig 2: A portion of the script used for the example web page shown in Figure 3 showing some of the tags of the proposed tag library being used with other HTML tags 
mobile browsers has earned substantial gain in performance in comparison to all earlier web content manipulation techniques for handheld devices, as no image compression technique is required to convert a large web image suitable for mobile display. A mobile browser has been developed to display the selected web content using the tags of the said tag library in a subtitle based hierarchical approach to improve the browsing experience to ensure effective mobile learning besides learning using desktop PCs. For implementation of the mobile browser, Sun Java Wireless Toolkit has been installed at the resource end and KXML parser has been used with J2ME application. Packaging with appropriate J2ME specification (MIDP and CLDC versions) the browser has been tested with wide range of Java enabled mobile phones. To get this service, user should have a Java enabled mobile with GPRS activated. The same algorithm can be used to develop a mobile browser as an Android application (.APK file) to be used in the Android devices. The present tag library can select and display, basically the textual data and images from the web content. The work can be extended to give tag based solutions for better display solutions of form elements, tabular data etc.

\section{REFERENCES}

[1] Liu, G. Z., \& Hwang, G. J. 2010. A key step to understanding paradigm shifts in e-learning: Towards context-aware ubiquitous learning. British Journal of Educational Technology, 41, 2, E1-E9.

[2] Dolittle, P., Lusk, D., Byrd, C. AND Mariano, G. 2009. iPods as Mobile Multimedia Learning Environments: Individual Differences and Instructional Design. Innovative Mobile Learning: Techniques and TEchnologies Hershey, PA: Information Science Reference, 83-101.

[3] Trifonova, A. \& Ronchetti, M. 2003. Where is Mobile Learning Going?, Proceedings of World Conference on E-Learning in Corporate, Government, Healthcare, and Higher Education, Chesapeake, VA, 1794-1801.

[4] Scornavacca, E., Huff, S. \& Marshall, S. 2008. Understanding the Value of Interactive SMS for Large Classes. Innovative Mobile Learning: Techniques and Technologies. H. Ryu and D. Parsons, eds., Information Science Reference, 48-59.

[5] Vahey, P., Tatar, D. \& Roschelle, J. 2004. Leveraging Handhelds to Increase Student Learning: Engaging Middle School Students with the Mathematics of Change. Proc. Sixth Int'1 Conf. Learning Sciences, 553560 .

[6] Naismith, L., Lonsdale, P., Vavoula, G. \& Sharples, M. 2004. Literature Review in Mobile Technologies and Learning. NESTA FutureLab, 11, 48.

[7] Sharples, M., Taylor, J. \& Vavoula, G. 2005. Towards a Theory of Mobile Learning. In H. van der Merwe \& T. Brown, Mobile technology: The future of learning in your hands, mLearn 2005 Book of Abstracts, 58.
[8] Garaj, V. 2010. m - Learning in the Education of Multimedia Technologists and Designers at the University Level: A User Requirements Study. IEEE Transactions on Learning Technologies, 3, 1, 24-32.

[9] Park, J., Parsons, D. \& Ryu, H. 2010. To Flow and Not to Freeze: Applying Flow Experience to Mobile Learning. IEEE Transactions on Learning Technologies, $3,1,56-67$.

[10] Traxler, J. 2007. Defining, Discussing, and Evaluating Mobile Learning: The moving finger writes and having writ.... International Review of Research in Open and Distance Learning. 8, 2, 1-12.

[11] Chen, C. H., Hwang, G. J., Yang, T. C., Chen, S. H. \& Huang, S. Y. 2009. Analysis of a ubiquitous performance support system for teachers. Innovations in Education and Teaching International, 46, 4, 421-433.

[12] Baluja S. 2006. Browsing on small screens: recasting web page segmentation into an efficient machine learning framework. Proceedings of the $15^{\text {th }}$ International Conference on World Wide Web, Edinburgh, Scotland, May 23-26, 33-42.

[13] Hwang,Y., KIM, J. \& SEO, E. 2003. A Structure-Aware Web Transcoding System for Mobile Devices. IEEE Computing, 7, 5, 14-21.

[14] Mahdavi, M., Khordadi, H. \& KHOOBKAR, M.H.2010. Web Transcoding for Mobile Devices Using a TagBased Technique. World Applied Sciences Journal 10 (Special Issue of Computer \& Electrical Engineering), 49-58.

[15] Rahman A. F. R., Alam H., Hartono R. AND Ariyoshi K. 2001. Automatic Summarization of Web Content to Smaller Display Devices. Proceedings of the 6th Document Analysis and Recognition Conference (ICDAR01), Seattle, USA, 10-13 September, 10641068.

[16] Berger A. \& Mittal, V. 2000. OCELOT: A System for Summarizing Web Pages. Proceedings of the 23rd Annual conference on Research and Development in Information Retrieval (ACM SIGIR), 144-151.

[17] Alam, H., Hartono R., Kumar A., Tarnikova Y., Rahman F. \& Wilcox C.(2003). Web Page Summarization for Handheld Devices: A Natural Language Approach. Proceedings of 7th International Conference on Document Analysis and Recognition (ICDAR'03), 2, 1153.

[18] Buyukkokten O., Garcia-Molina, H. \& Paepcke, A. 2001. Text Summarization of Web pages on Handheld Devices. Proceedings of 10th International Conference on World Wide Web, Hong Kong, 652-662. 


\section{Sub Title Based Content Presentation}

\section{Content subtitle 1}

This is paragapd 1 for web. . This part of the paragraph has been selected for mobde display from para 1 usder subtitle 1 .... para 1 coetinued.

This is paragapd 2 for web... This part of the paragaph has beta stlected for moble diplay from para 2 under abeate 1 .... para 2 coetiroed.

\section{(*)}

\section{WELCOME!}

Content subtitle 2 only for web and not for mobile

This is paragaph 1 under subtele 2

\section{Content subtitle 3}

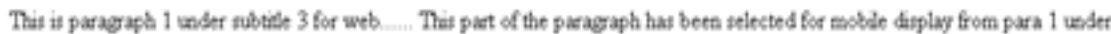
subthe 3 Here is an mage with etebeded teat in 2

$\$$ intenet

Fig 3: The example web page

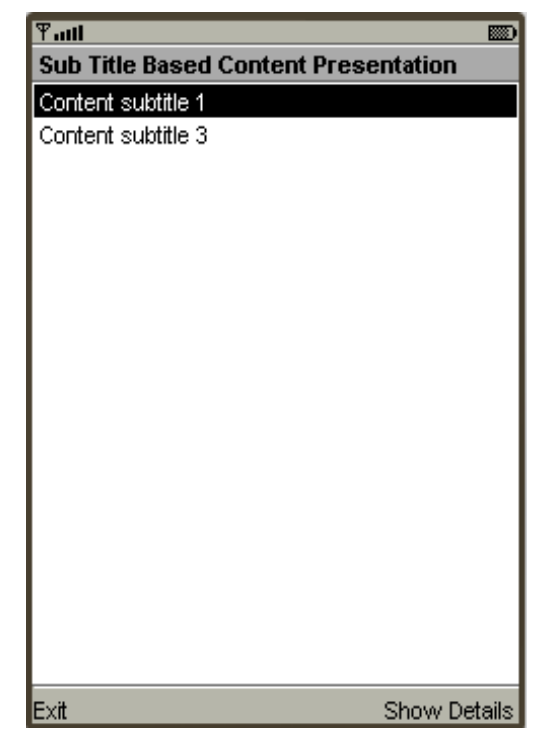

Fig 4: Subtitle 1 is selected

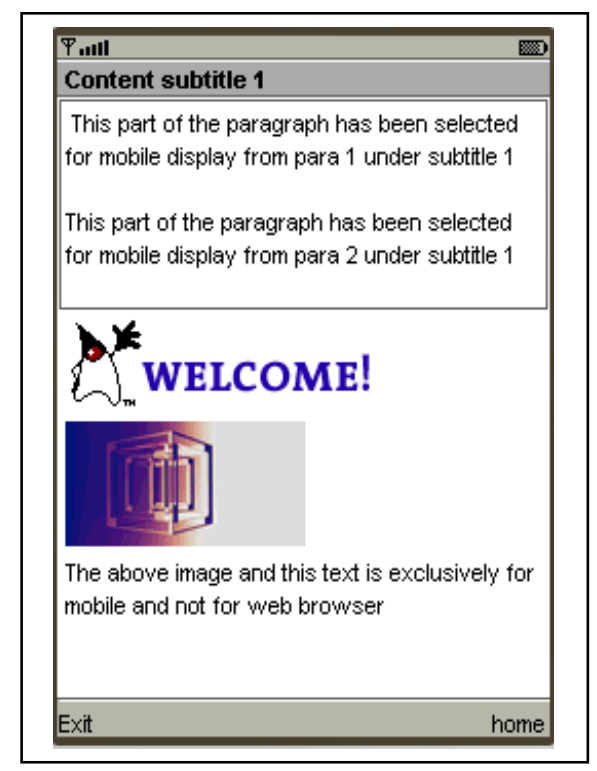

Fig 5: Content under subtitle 1 


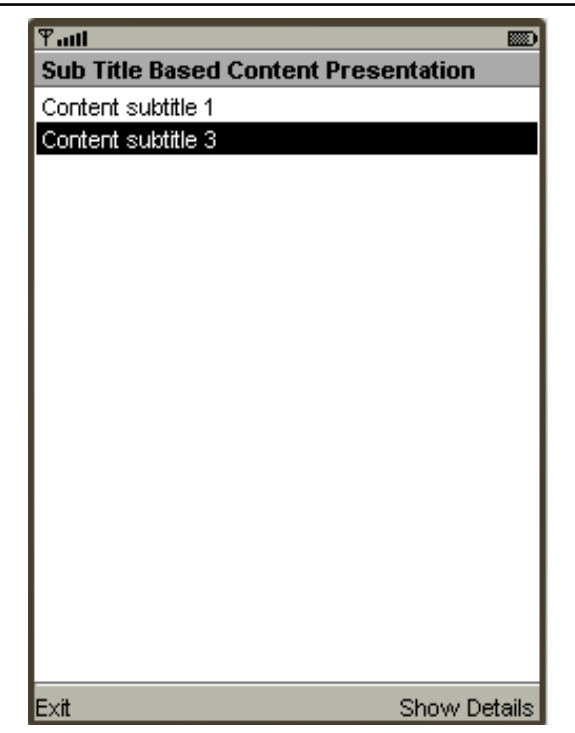

Fig. 6: Subtitle 3 is selected

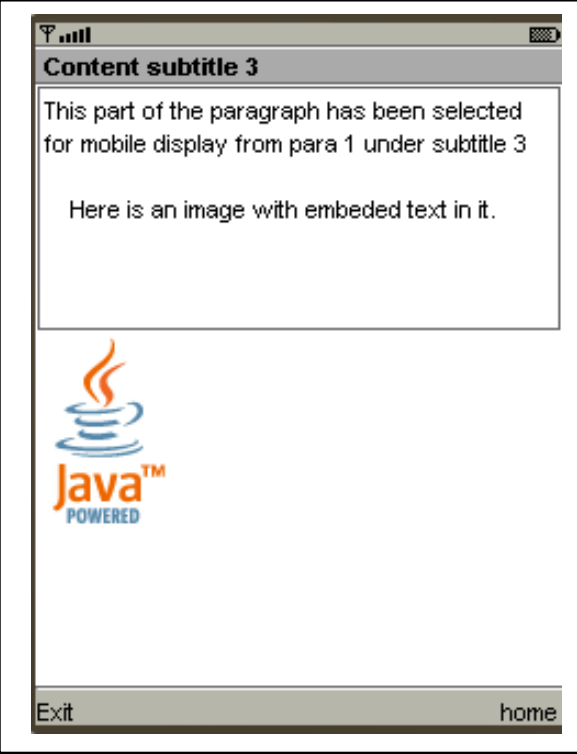

Fig. 7: Content under subtitle 3 